\title{
OPEN Tradeoffs between dispersal and reproduction at an invasion front of cane toads in tropical Australia
}

\author{
Crystal Kelehear $\mathbb{1}^{1,2}$ \& Richard Shine $\mathbb{C}^{1,3^{*}}$ \\ Individuals at the leading edge of a biological invasion experience novel evolutionary pressures on \\ mating systems, due to low population densities coupled with tradeoffs between reproduction and \\ dispersal. Our dissections of $>1,200$ field-collected cane toads (Rhinella marina) at a site in tropical \\ Australia reveal rapid changes in morphological and reproductive traits over a three-year period \\ after the invaders first arrived. As predicted, individuals with dispersal-enhancing traits (longer \\ legs, narrower heads) had reduced reproductive investment (lower gonad mass). Post-invasion, the \\ population was increasingly dominated by individuals with less dispersive phenotypes and a higher \\ investment into reproduction (including, increased expression of sexually dimorphic traits in males). \\ These rapid shifts in morphology and reproductive biology emphasise the impacts of the invasion \\ process on multiple, interlinked aspects of organismal biology.
}

Any organism has a finite store of resources to allocate among competing demands such as maintenance, growth, and reproduction; and hence, natural selection is expected to fine-tune that allocation in ways that maximise lifetime reproductive success ${ }^{1}$. As a result, levels of investment into reproduction should depend upon competing priorities, with organisms decreasing reproductive output if investment into other functions yields higher benefits in fitness. Such tradeoffs may be especially clear during biological invasions, because of differences in evolutionary forces at the invasion front versus in long-colonised areas $^{2}$. Individuals at an expanding range edge often exhibit unusually high rates of dispersal ${ }^{3,4}$, requiring energy that may decrease allocation of resources to other functions such as immune defence $e^{5-7}$ and reproduction ${ }^{8-11}$. The increased allocation to dispersal is exacerbated by the non-adaptive process of spatial sorting; individuals that allocate more energy into dispersal will likely be close to the range edge, even if more rapid dispersal does not enhance fitness ${ }^{12}$.

To test these ideas, we need to compare attributes of individuals at an expanding invasion front to conspecifics in longer-colonised areas. We can perform that comparison either through space (monitoring sites with different times since invasion) or through time (monitoring a single site as an invasion passes through). Most available evidence comes from spatial comparisons, which minimise confounding due to temporal (seasonal, annual) variation but introduce confounding factors that vary among sites (e.g. resource levels, predator abundance) ${ }^{13}$. Comparisons through time at a single site avoid those problems, but require longer-term monitoring and introduce confounds associated with temporally variable factors. In the current paper, we describe the results of studies over a three-year period at a single site, beginning soon after the initial arrival of a colonising species.

The invasion of cane toads (Rhinella marina, formerly Bufo marinus) through tropical Australia has attracted considerable research. Individuals in the vanguard disperse long distances every night, and exhibit phenotypic traits (such as longer legs and narrower heads) that facilitate sustained locomotion ${ }^{14-19}$. Spatial comparisons among toad populations suggest a tradeoff between dispersal rate and reproductive output: compared to conspecifics from long-colonised areas, females from invasion-front populations reproduce at low rates even under standardised conditions ${ }^{20}$, and males have smaller testes relative to body size ${ }^{21}$. We sampled toads at a single site through a three-year span to look for the same kinds of changes through time, and for predicted tradeoffs between dispersal and reproduction.

\footnotetext{
${ }^{1}$ School of Life and Environmental Sciences, University of Sydney, Sydney, NSW, 2006, Australia. ${ }^{2}$ Department of Biology, Geology \& Physical Sciences, Sul Ross State University, Alpine, Texas, 79832, USA. ${ }^{3}$ Department of Biological Sciences, Macquarie University, Sydney, NSW, 2109, Australia. *email: rick.shine@mq.edu.au
} 


\begin{tabular}{|l|l|l|l|}
\hline \multirow{4}{*}{ Sample size } & Year 1 & Year 2 & Year 3 \\
\cline { 2 - 4 } & 719 & 443 & 110 \\
\hline Morphological traits & \multicolumn{5}{l}{} \\
\hline Snout-urostyle length (mm) & $100.82(0.48)$ & $103.49(0.62)$ & $104.22(1.24)$ \\
\hline Body mass (g) & $128.98(2.19)$ & $135.37(2.79)$ & $145.06(5.63)$ \\
\hline Head width (mm) & $41.10(0.20)$ & $42.48(0.26)$ & $42.63(0.52)$ \\
\hline Tibia length (mm) & $40.92(0.19)$ & $41.55(0.25)$ & $41.86(0.49)$ \\
\hline Sex ratio (\% male) & 60.22 & 60.95 & 78.90 \\
\hline Reproductive traits & $0.20(0.006)$ & $0.25(0.009)$ & $0.29(0.019)$ \\
\hline Total testes mass (g) & $5.89(0.68)$ & $7.53(0.99)$ & $7.91(3.05)$ \\
\hline Total ovary mass (g) & \multicolumn{5}{l}{} \\
\hline Rate of change (haldanes) & \multicolumn{5}{l|}{} \\
\hline Relative head width (all) & & 0.23 & -0.10 \\
\hline Relative head width (male) & & 0.27 & -0.08 \\
\hline Relative head width (female) & & 0.18 & -0.02 \\
\hline Relative tibia length (all) & & -0.21 & 0.02 \\
\hline Relative tibia length (male) & & -0.04 & -0.05 \\
\hline Relative tibia length (female) & & -0.49 & -0.27 \\
\hline Relative testes mass & & 0.32 & -0.55 \\
\hline Relative ovary mass & & 0.11 & -0.14 \\
\hline
\end{tabular}

Table 1. Annual cane toad sample sizes and trait values. Table shows mean values and associated SEs for data from each year of the study. "Relative" variables are residual scores from the linear regression of that trait on snout-urostyle length (linear traits) or body mass (mass-based traits).

\section{Results}

Mean body sizes remained similar across the sampling period (Table 1), but there were significant shifts among years in several traits. In male toads, testes mass relative to body mass increased from Year 1 to Year 2 (significant with a posthoc test), then declined in Year 3 (ANOVA, $F_{2,655}=8.52, P<0.0002$; Fig. 1a). Changes in other sexually dimorphic traits were more straightforward, with mean values increasing through time for skin rugosity $\left(F_{2,718}=17.97, P<0.0001\right.$; posthoc $1<2<3$; Fig. 1 b), skin colour $\left(F_{2,719}=61.11, P<0.0001\right.$; posthoc $1<2<3$; Fig. 1c) and development of the metatarsal tubercle $\left(F_{2,718}=21.82, P<0.0001\right.$; posthoc $1<2<3$; Fig. $\left.1 \mathrm{~d}\right)$. In females, ovary mass relative to body mass did not change significantly among years $\left(F_{2,424}=0.59, P=0.56\right)$.

Changes through time were also evident for morphological traits associated with enhanced rates of dispersal, and known to differ between males and females ${ }^{17,18}$. Tibia lengths relative to body length declined rapidly post-invasion in female toads, but not males (interaction year*sex, $F_{2,718}=17.97, P<0.0001$; posthoc $1<2<3$; $F_{2,1270}=7.92, P<0.0005$; Fig. $\left.2 \mathrm{a}\right)$. In contrast, relative head widths increased from Year 1 to Year 2 in both sexes (main effect of year, $F_{2,1270}=6.72, P<0.0015$; sex effect, $F_{1,1270}=5.26, P<0.022$; interaction year*sex, $F_{2,1270}=0.08, P=0.92$; Fig. 2b).

Rates of change in quantitative traits can be expressed in Haldanes (one Haldane = a change of one standard deviation per generation ${ }^{22}$ ). Cane toads mature at approximately one year of age ${ }^{23}$, so annual rates of change equate to Haldanes. Rates of annual change in the traits that we measured ranged from -0.46 to +0.32 Haldanes between Years 1 and 2, and from -0.55 to -0.05 Haldanes between Years 2 and 3, with no obvious differences between sexes or between morphological versus reproductive traits (Table 1).

Lastly, variation in gonad mass relative to body mass among individuals was negatively (albeit weakly) associated with variation in relative tibia length in both sexes (males $n=656, r^{2}=0.008, P<0.025$; females $n=425$, $\left.r^{2}=0.016, P<0.01\right)$. Variation in relative head width was negatively associated with variation in relative gonad mass in females $\left(n=425, r^{2}=0.022, P<0.002\right)$ but not in males $\left(n=656, r^{2}=0.0003, P=0.68\right)$. If we include Year \# as well as the morphological variables in an ANCOVA, the link between relative head width and relative gonad mass is strengthened in females $\left(F_{1,421}=10.31, P<0.0015\right)$ whereas the same relationship in males remains non-significant $\left(F_{1,655}=0.01, P=0.92\right)$. The same result is obtained with analyses including Year \# as well as relative tibia length (tibia length effect for males, $F_{1,655}=2.90, P=0.09$; tibia length effect for females, $F_{1,421}=5.97$, $P<0.015)$. These results imply that the negative correlation between variables related to dispersal versus reproduction in females was not a result of combining data for years with different mean values for each of these traits. Looking only at Year 1 (closest to the invasion front, and with the largest sample size), females with larger relative ovary masses had narrower heads $\left(F_{1,277}=13.32, P<0.0003\right)$ and shorter legs $\left(F_{1,277}=9.89, P<0.002\right)$.

\section{Discussion}

When we first began sampling cane toads, a few years after they first arrived at our study site in tropical Australia, the population was dominated by individuals with dispersal-enhancing phenotypes (long legs, narrow heads ${ }^{17-19}$ ). Males at the invasion vanguard exhibited low gonad masses, and minimal development of sexually dimorphic traits. Over the next three years, those attributes shifted; newly-arriving toads showed phenotypic traits less strongly associated with dispersal (e.g., had shorter limbs), had larger testes (at least briefly), and displayed more highly-developed secondary sexual characteristics (e.g., skin rugosity). Within the overall sample, phenotypic 

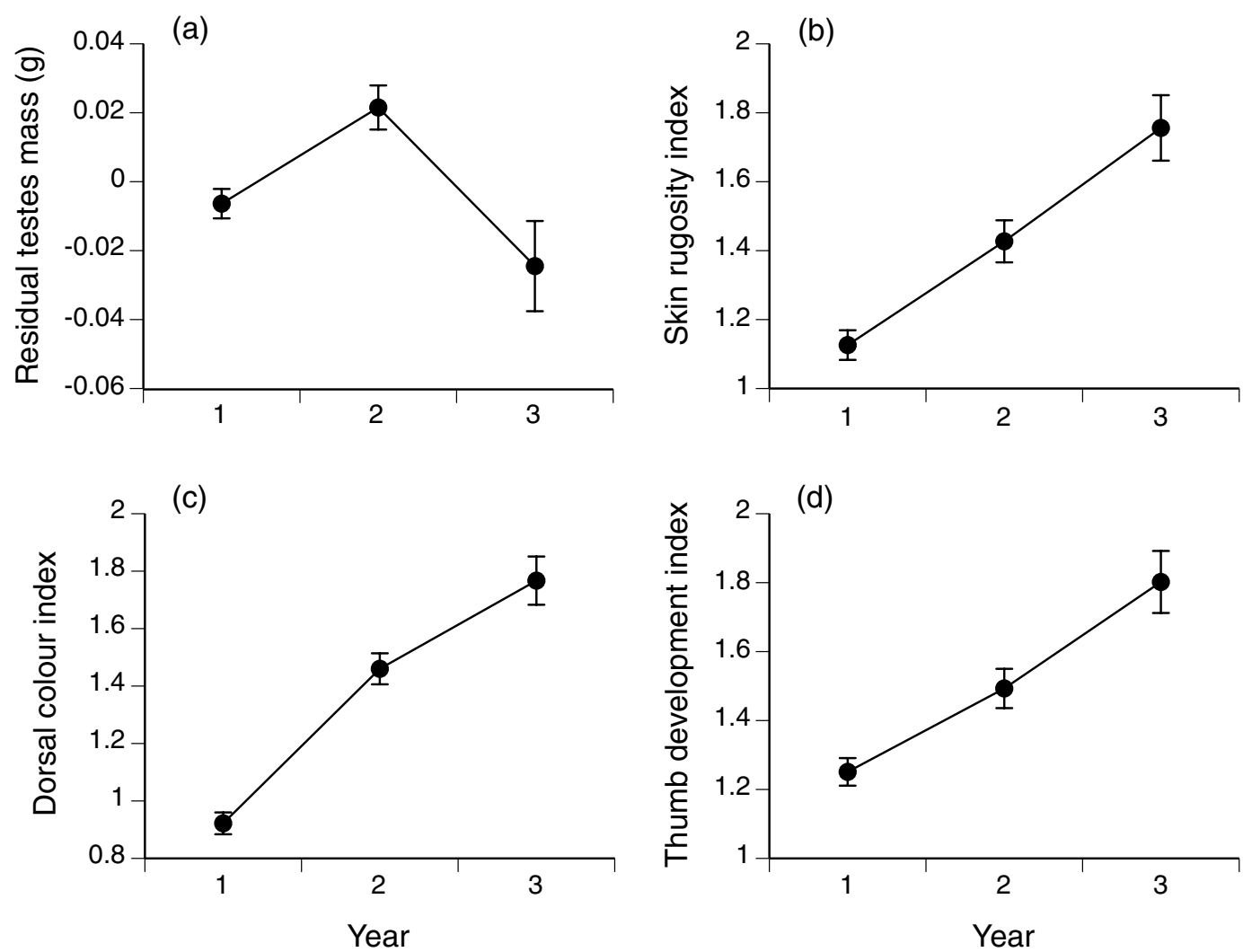

Figure 1. Variation in morphological traits of male cane toads (Rhinella marina) as a function of time since initial invasion.

traits associated with dispersal were negatively correlated with our measures of reproductive output. The reduction in tibia length post-colonisation was more rapid in female toads than in males, such that the degree of sexual dimorphism in this trait increased through time (Fig. 2a).

Although annual changes in relative ovary mass were not statistically significant, this result may tell us little because a female toad may have a small ovary either because she is non-reproductive or because she has recently spawned. Hence, ovary mass may not reflect reproductive rate. In contrast, testes masses may better reflect reproductive condition; and spatial comparisons have reported smaller testes in invasion-front toads than in range-core toads ${ }^{21}$. At a quantitative level, the rates of change in trait values per generation (in Haldanes) fell within the range reported in other studies of microevolutionary rates of characteristics under intense selection ${ }^{22}$.

In summary, concurrent changes in dispersal-relevant morphology and in reproductive investment accord well with predictions from theoretical models about post-invasion attenuation in dispersal capacity. Specifically, our data reveal the pattern expected from the predicted tradeoff between allocation of energy to dispersal versus to life-history functions ${ }^{2}$. Spatial sorting (accumulation of dispersal-enhancing phenotype at the expanding range edge) likely plays a role also, and the net phenotypic shifts through time likely reflect a combination of life-history tradeoffs and spatial sorting ${ }^{2,12,15}$. For males, the fitness disadvantages of lowered investment into reproduction may be minimal at the invasion front, where competitors are scarce; but those disadvantages rapidly increase post-invasion, with the rise in population densities (and hence, intensity of male-male competition). Operational sex ratios became more male-biased also (see Table 1), further increasing the intensity of male-male competition and hence, imposing increasing disadvantages to males with low investment into reproductive activities.

In general, our results from phenotypic changes through time are consistent with inferences based on comparisons through space. For example, a narrower head and longer legs have previously been reported in invasion-front cane toads $s^{17,18}$. Perhaps the most striking aspect of our results is the timescale of such changes. Spatial comparisons generally have compared populations with time-since-colonisation intervals at decadal or longer scales, whereas we saw rapid changes within a three-year period. Consistent with our results, temporal analyses reported similarly rapid shifts in energy balance ${ }^{24}$ and dispersal behaviour ${ }^{15}$ of toads at our study site post-invasion. Lacking data on attributes of offspring raised in common-garden conditions, we cannot distinguish whether these changes are driven by phenotypic plasticity or by adaptation.

One intriguing pattern in our data is the rapid change in sexual dimorphism in a morphological trait (relative limb length) that is highly correlated with dispersal rate and has shifted over the course of the toad invasion (longer legs at the invasion front ${ }^{14,18}$ ). Mathematical models predict that if one sex is intrinsically faster than the other, the fitness benefit to more rapid dispersal at the invasion front will be weaker in the more rapidly-dispersing sex (who otherwise will outpace the slower $\operatorname{sex}^{25}$ ). Male cane toads have longer legs than females, and hence are faster dispersers ${ }^{18}$. Thus, the disproportionate advantage to higher-than-usual rates of 

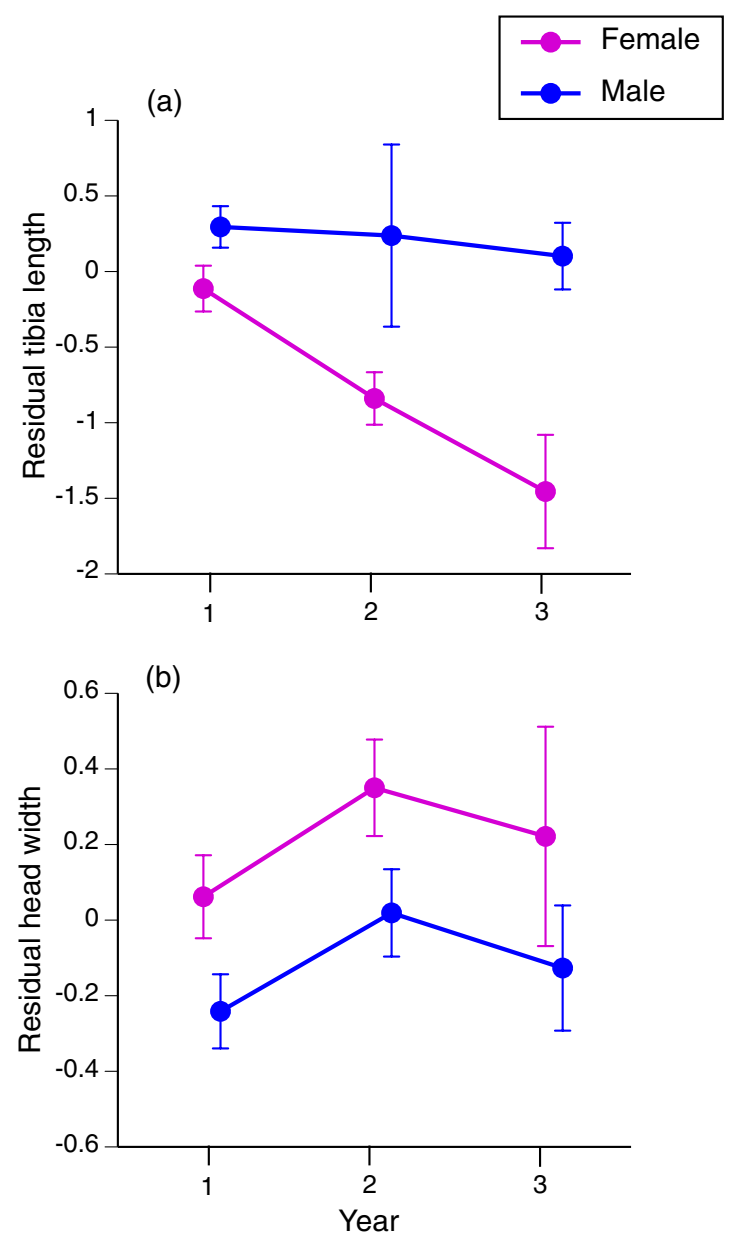

Figure 2. Sexual dimorphism in cane toads (Rhinella marina) as a function of time since initial invasion.

dispersal (=longer-than-usual legs) applies only to females at the invasion front. As soon as toads colonise an area, operational sex ratios no longer depend on sex differences in rate of dispersal. The advantage of unusually fast dispersal to females (relative to males) thus declines, resulting in a rapid decline in limb lengths in the slower sex (females); and hence, a shift in sexual dimorphism for this trait (see Fig. 2a).

Tradeoffs between dispersal ability and reproductive investment have been documented in many species, but involve a variety of proximate mechanisms. The simplest is for reduced reproductive investment to directly enhance dispersal rate: for example, lighter seeds can float further on the wind ${ }^{26}$ and female reptiles carrying a heavier clutch cannot run as quickly ${ }^{27}$. The tradeoff between flight and fecundity has been well-studied in insects ${ }^{28,29}$. However, tradeoffs are evident also at the genetic level: for example, artificial selection on fecundity generated rapid changes in wing morphology in crickets ${ }^{8}$. The tradeoff we have documented in cane toads also involves heritable traits (see above) and hence, is plausibly due to variation among individuals in allocation to competing functions. Individuals at the invasion front have evolved to disperse rapidly, via natural selection (access to abundant food at the front ${ }^{24}$ ), sexual selection (lowered male-male competition at the front ${ }^{30}$ ) and spatial sorting (non-adaptive winnowing of dispersal-enhancing genes across the invasion history ${ }^{12}$ ). These individuals exhibit morphological traits that enhance dispersal ability, with a commensurate decrease in investment into competing functions such as immune responses ${ }^{6}$ and reproduction ${ }^{20,21}$ (and current paper). As soon as the front passes, however, increasing population densities and attenuation of the advantages to dispersal may shift selective forces to favour phenotypes with less emphasis on dispersal relative to other functions ${ }^{31}$. Our data suggest that such transitions may occur very quickly, such that the distinctive phenotypes of individuals in the invasion vanguard are rapidly replaced by traits better-suited to the evolutionary forces at play after the system achieves spatial equilibrium.

\section{Methods}

Study site and species. We collected toads within Australia's wet-dry tropics, centred on a site $60 \mathrm{~km}$ east of the city of Darwin $\left(12^{\circ} 34^{\prime} 43.54^{\prime \prime} \mathrm{S}, 131^{\circ} 18^{\prime} 51.55^{\prime \prime} \mathrm{E}\right)$. Higher ground is dominated by savanna woodland, with extensive floodplains in low-lying areas. The area is hot year-round, with monsoonal rains from January to March in most years ${ }^{24}$.

Extensive research, based primarily on spatial sampling across the toad's invaded range, has documented many differences between individuals from the invasion front versus range-core, and common-garden 
breeding experiments have confirmed that many of those phenotypic differences are heritable ${ }^{6,19}$. For example, the offspring of invasion-front individuals exhibit higher rates of dispersal, and more consistent directionality of movement ${ }^{23,32}$. Many of the evolved differences involve traits that enhance rates of dispersal; for example, invasion-front individuals have higher endurance ${ }^{33}$ across a wider range of abiotic conditions ${ }^{34}$, longer legs ${ }^{14,18}$, narrower heads ${ }^{17}$, and invest less energy into immune function ${ }^{6,35}$ and reproduction ${ }^{20,21}$. Invasion-front individuals also tend to be bolder and more active ${ }^{36,37}$. Some of the geographically divergent traits are highly heritable, whereas others are influenced by phenotypic plasticity also ${ }^{19,23,38}$.

Sampling methods. Our sampling commenced in 2008 , three years after toads first arrived ${ }^{39}$, and continued for three further years (see Supplementary Information). Population densities of toads increased each year during that period ${ }^{40,41}$, suggesting that the invasion front was still passing through the area and hence, the population should be subject to both selective tradeoffs and spatial sorting. Toad abundance then decreased in 2012 and thereafter ${ }^{39}$. We defined Year 1 as September 2008 to August 2009, Year 2 as September 2009 to August 2010, and Year 3 as October 2010 to June 2011. Thus, each year's sample included the wet-season period during which toads actively disperse ${ }^{42}$. The abundance of toads was not significantly correlated with annual variation in precipitation over the period 2005 to $2012^{39}$, suggesting that our temporal comparisons were not strongly affected by variation in weather conditions. Extensive mark-recapture studies at our main study site detected no toads staying more than a single year; thus, all of the sampled animals likely arrived at their respective locations in the same year that they were collected. We hand-captured adult toads while they were active at night ${ }^{43}$. The toads were humanely killed and dissected the following day. We recorded body size (snout-urostyle length [=SUL], body mass), head width, and length of the tibia. We removed gonads, blotted them dry, and weighed them to $0.001 \mathrm{~g}$ (Precision Balance FX-200i WP, A\&D Company Limited, Tokyo, Japan). For males, we also scored the degree of development of sexually dimorphic traits on a 3-point scale for each of three variables; sexually active males develop more rugose skin, yellow dorsal colouration, and enlarged metatarsal tubercles on the thumbs ${ }^{43}$.

This work was approved by the University of Sydney Animal Ethics Committee (L04/1-2010/3/5193; L04/52010/2/5334), and all methods were performed in accordance with the relevant guidelines and regulations.

Data were checked for normality and variance homogeneity prior to analysis; no transformations were needed. Using JMP 13.0, we analysed temporal shifts in traits via ANOVA, using Year \# $(1,2,3)$ as a factor and phenotypic traits as dependent variables. Because testes mass increases with overall body mass, we used residual scores from the general linear regression of gonad mass on body mass as our measure of gonad size (calculated separately in the two sexes). To examine temporal changes in sexually dimorphic traits (relative tibia length and relative head width), we used Year \# and sex as factors, and the morphological feature (residual score from the linear regression of that trait against SUL) as the dependent variable. To assess predicted tradeoffs, we calculated Pearson correlations between morphological and reproductive traits. To see if such correlations were confounded by annual changes in trait values, we also conducted ANCOVAs with year as factor, morphological trait (e.g. residual tibia length) as covariate, and relative gonad mass as the dependent variable. Non-significant interaction terms were deleted and main effects recalculated. We used Tukey post-hoc tests to locate significant differences revealed by ANOVAs and ANCOVAs.

Received: 28 October 2019; Accepted: 29 December 2019;

Published online: 16 January 2020

\section{References}

1. Bonte, D. \& Dahirel, M. Dispersal: a central and independent trait in life history. Oikos 126, 472-479 (2017).

2. Perkins, T. A., Boettiger, C. \& Phillips, B. L. After the games are over: life-history trade-offs drive dispersal attenuation following range expansion. Ecol. Evol. 6, 6425-6434 (2016).

3. Lombaert, E. et al. Rapid increase in dispersal during range expansion in the invasive ladybird Harmonia axyridis. J. Evol. Biol. 27, 508-517 (2014).

4. Courant, J., Secondi, J., Guillemet, L., Vollette, E. \& Herrel, A. Rapid changes in dispersal on a small spatial scale at the range edge of an expanding population. Evol. Ecol. 33, 1-14 (2019).

5. Lee, K. A. \& Klasing, K. C. A role for immunology in invasion biology. Trends Ecol. Evol. 19, 523-529 (2004),

6. Llewellyn, D., Thompson, M. B., Brown, G. P., Phillips, B. L. \& Shine, R. Reduced investment in immune function in invasion-front populations of the cane toad (Rhinella marina) in Australia. Biol. Invasions 14, 999-1008 (2012).

7. Selechnik, D. et al. Effects of invasion history on physiological responses to immune system activation in invasive Australian cane toads. PeerJ 5, e3856 (2017).

8. Roff, D. A., Tucker, J., Stirling, G. \& Fairbairn, D. J. The evolution of threshold traits: effects of selection on fecundity and correlated response in wing dimorphism in the sand cricket. J. Evol. Biol. 12, 535-546 (1999).

9. Gibbs, M., Breuker, C. J., Hesketh, H., Hails, R. S. \& Van Dyck, H. Maternal effects, flight versus fecundity trade-offs, and offspring immune defence in the Speckled Wood butterfly, Pararge aegeria. BMC Evol. Biol. 10, 345 (2010).

10. Gibbs, M., Breuker, C. J. \& Van Dyck, H. Flight during oviposition reduces maternal egg provisioning and influences offspring development in Pararge aegeria (L.). Physiol. Entomol. 35, 29-39 (2010).

11. Helms, J. A. \& Kaspari, M. Reproduction-dispersal tradeoffs in ant queens. Insectes sociaux 62, 171-181 (2015).

12. Shine, R., Brown, G. P. \& Phillips, B. L. An evolutionary process that assembles phenotypes through space rather than through time. Proc. Natl Acad. Sci. USA 108, 5708-5711 (2011).

13. Phillips, B. L., Brown, G. P., Travis, J. M. J. \& Shine, R. Reid's paradox revisited: the evolution of dispersal kernels during range expansion. Am. Nat. 172, S34-S48 (2008).

14. Phillips, B. L., Brown, G. P., Webb, J. K. \& Shine, R. Invasion and the evolution of speed in toads. Nature 439, 803 (2006).

15. Lindström, T., Brown, G. P., Sisson, S. A., Phillips, B. L. \& Shine, R. Rapid shifts in dispersal behavior on an expanding range edge. Proc. Natl Acad. Sci. USA 110, 13452-13456 (2013).

16. Pizzatto, L., Both, C., Brown, G. \& Shine, R. The accelerating invasion: dispersal rates of cane toads at an invasion front compared to an already-colonized location. Evol. Ecol. 31, 533-545 (2017).

17. Hudson, C. M., McCurry, M. R., Lundgren, P., McHenry, C. R. \& Shine, R. Constructing an invasion machine: the rapid evolution of a dispersal-enhancing phenotype during the cane toad invasion of Australia. PLoS One 11, e0156950 (2016). 
18. Hudson, C. M., Brown, G. P. \& Shine, R. It is lonely at the front: contrasting evolutionary trajectories in male and female invaders. R. Soc. Open Sci. 3, 160687 (2016).

19. Hudson, C., Brown, G. P., Stuart, K. \& Shine, R. Sexual and geographic divergence in head widths of invasive cane toads, Rhinella marina (Anura: Bufonidae) is driven by both rapid evolution and plasticity. Biol. J. Linn. Soc. 124, 188-199 (2018).

20. Hudson, C. H., Phillips, B. L., Brown, G. P. \& Shine, R. Virgins in the vanguard: low reproductive frequency in invasion-front cane toads. Biol. J. Linn. Soc. 116, 743-747 (2015).

21. Friesen, C. R. \& Shine, R. At the invasion front, male cane toads (Rhinella marina) have smaller testes. Biol. Lett. 15, 20190339 (2019).

22. Hendry, A. P. \& Kinnison, M. T. Perspective: the pace of modern life: measuring rates of contemporary microevolution. Evolution 53, 1637-1653 (1999).

23. Brown, G. P., Phillips, B. L. \& Shine, R. The straight and narrow path: the evolution of straight-line dispersal at a cane toad invasion front. Proc. R. Soc. B 281, 20141385 (2014).

24. Brown, G., Kelehear, C. \& Shine, R. The early toad gets the worm: cane toads at an invasion front benefit from higher prey availability. J. Anim. Ecol. 82, 854-862 (2013).

25. Meier, C. M., Starrfelt, J. \& Kokko, H. Mate limitation causes sexes to coevolve towards more similar dispersal kernels. Oikos 120, 1459-1468 (2011).

26. Tabassum, S. \& Leishman, M. R. Have your cake and eat it too: greater dispersal ability and faster germination towards range edges of an invasive plant species in eastern Australia. Biol. Invasions 20, 1199-1210 (2018).

27. Shine, R. Effects of pregnancy on locomotor performance: an experimental study on lizards. Oecologia 136, 450-456 (2003).

28. Hughes, C. L., Dytham, C. \& Hill, J. K. Evolutionary trade-offs between reproduction and dispersal in populations at expanding range boundaries. Proc. R. Soc. B 270, S147-S150 (2003).

29. Duthie, A. B., Abbott, K. C. \& Nason, J. D. Trade-offs and coexistence in fluctuating environments: evidence for a key dispersal fecundity trade-off in five non-pollinating fig wasps. Am. Nat. 186, 151-158 (2015).

30. Clarke, G. S., Shine, R. \& Phillips, B. L. May the (selective) force be with you: spatial sorting and natural selection exert opposing forces on limb length in an invasive amphibian. J. Evol. Biol. 32, 994-1001 (2019).

31. Duckworth, R. A. \& Badyaev, A. V. Coupling of dispersal and aggression facilitates the rapid range expansion of a passerine bird. Proc. Natl Acad. Sci. USA 104, 15017-15022 (2007).

32. Phillips, B. L., Brown, G. P. \& Shine, R. Evolutionarily accelerated invasions: the rate of dispersal evolves upwards during the range advance of cane toads. J. Evol. Biol. 23, 2595-2601 (2010).

33. Llewelyn, J., Phillips, B. L., Alford, R. A., Schwarzkopf, L. \& Shine, R. Locomotor performance in an invasive species: cane toads from the invasion front have greater endurance, but not speed, compared to conspecifics from a long-colonised area. Oecologia 162, 343-348 (2010)

34. Kosmala, G., Christian, K., Brown, G. \& Shine, R. Locomotor performance of cane toads differs between native-range and invasive populations. R. Soc. Open Sci. 4, 170517 (2017)

35. Brown, G. P., Kelehear, C., Shilton, C. M., Phillips, B. L. \& Shine, R. Stress and immunity at the invasion front: a comparison across cane toad (Rhinella marina) populations. Biol. J. Linn. Soc. 116, 748-760 (2015).

36. Gruber, J., Brown, G., Whiting, M. J. \& Shine, R. Geographic divergence in dispersal-related behaviour in cane toads from rangefront versus range-core populations in Australia. Behav. Ecol. Sociobiol. 71, 38 (2017).

37. Gruber, J., Brown, G., Whiting, M. J. \& Shine, R. Is the behavioural divergence between range-core and range-edge populations of cane toads (Rhinella marina) due to evolutionary change or developmental plasticity? R. Soc. Open Sci. 4, 170789 (2017).

38. Stuart, K., Shine, R. \& Brown, G. P. Proximate mechanisms underlying the rapid modification of phenotypic traits in cane toads (Rhinella marina) across their invasive range within Australia. Biol. J. Linn. Soc. 126, 68-79 (2019).

39. Brown, G. P. \& Shine, R. Using a natural population collapse of an invasive species to assess the benefits of invader control for native species. Biol. Invasions 21, 2781-2788 (2019).

40. Phillips, B. L., Greenlees, M. J., Brown, G. P. \& Shine, R. Predator behaviour and morphology mediates the impact of an invasive species: cane toads and death adders in Australia. Anim. Conserv. 13, 53-59 (2010).

41. Brown, G. P., Phillips, B. L. \& Shine, R. The ecological impact of invasive cane toads on tropical snakes: field data do not support predictions from laboratory studies. Ecology 92, 422-431 (2011).

42. Brown, G. P., Kelehear, C. \& Shine, R. Effects of seasonal aridity on the ecology and behaviour of invasive cane toads in the Australian wet-dry tropics. Funct. Ecol. 25, 1339-1347 (2011).

43. Kelehear, C. \& Shine, R. Non-reproductive male cane toads (Rhinella marina) withhold sex-identifying information from their rivals. Biol. Lett. 15, 20190462 (2019).

\section{Acknowledgements}

We thank Greg Brown for advice and assistance, Ben Phillips for comments on the manuscript, and the Australian Research Council for funding. We are grateful to the staff of Beatrice Hill farm and the Darwin RAAF golf course for site access, and the Northern Territory Land Corporation for logistical support. This work was approved by the University of Sydney Animal Ethics Committee (L04/1-2010/3/5193; L04/5-2010/2/5334).

\section{Author contributions}

C.K. gathered the data, R.S. and C.K. analysed the data, R.S. and C.K. jointly wrote the manuscript.

\section{Competing interests}

The authors declare no competing interests.

\section{Additional information}

Supplementary information is available for this paper at https://doi.org/10.1038/s41598-019-57391-x.

Correspondence and requests for materials should be addressed to R.S.

Reprints and permissions information is available at www.nature.com/reprints.

Publisher's note Springer Nature remains neutral with regard to jurisdictional claims in published maps and institutional affiliations. 
(c) (i) Open Access This article is licensed under a Creative Commons Attribution 4.0 International License, which permits use, sharing, adaptation, distribution and reproduction in any medium or format, as long as you give appropriate credit to the original author(s) and the source, provide a link to the Creative Commons license, and indicate if changes were made. The images or other third party material in this article are included in the article's Creative Commons license, unless indicated otherwise in a credit line to the material. If material is not included in the article's Creative Commons license and your intended use is not permitted by statutory regulation or exceeds the permitted use, you will need to obtain permission directly from the copyright holder. To view a copy of this license, visit http://creativecommons.org/licenses/by/4.0/.

(c) The Author(s) 2020 